\title{
Epimedium protects against dyszoospermia in mice with Pex3 knockout by exerting antioxidant effects and regulating the expression level of P16
}

\author{
Haiyang Zhao (iD ${ }^{1,6}$, Tingting Zhao ${ }^{2,6}$, Jihong Yang ${ }^{3}$, Qianqian Huang ${ }^{3}$, Hua Wu ${ }^{3}$, Yueyun Pan ${ }^{4}$, Hui Wang (iD ${ }^{1,5 凶}$ and Yun Qian (iD ${ }^{3 凶}$
}

(c) The Author(s) 2022

Oxidative stress (OS) is one of the primary factors leading to male infertility. Oral administration of antioxidants has thus far been found to significantly improve the quality of human sperm. Therefore, antioxidant treatment has become the consensus among international experts on male infertility. In this study, peroxisomal biogenesis factor 3 (Pex3)-knockout (KO, -/-) mice were used as a model to compare the efficacy of three types of traditional Chinese medicine (TCM) granules (Epimedium [YYH], Cuscuta [TSZ], and Rhodiola [HJT]) for male reproductive function rescue. YYH was revealed to be the best and exerted a rescue effect on Pex3-/mice with spermatogenesis defects. In addition, YYH prominently reduced ROS levels in the testes, inhibited DNA oxidative damage in spermatogenic cells, promoted the proliferation of spermatogenic cells, and inhibited apoptosis in Pex3-/- male mice. Furthermore, the mechanism by which YYH ameliorated dyszoospermia was confirmed via the establishment of cyclin-dependent kinase inhibitor 2 A (P16Ink4a)-KO mice. Specifically, Pex3-/- mice produced elevated amounts of ROS, which damaged germ cell DNA and further activated the signaling pathway of the cell senescence regulatory protein P16-CDK6, resulting in cell cycle arrest and eventually contributing to spermatogenesis dysfunction. YYH supplementation partially corrected the associated phenotype in gene KO mice by affecting P16 expression levels, thus improving the reproductive outcome to a certain extent.

Cell Death and Disease (2022)13:69; https://doi.org/10.1038/s41419-021-04435-8

\section{INTRODUCTION}

Infertility is a global issue. WHO estimates that $10-15 \%$ of childbearing couples are infertile, and $50 \%$ of infertility cases are caused by male factors. Among infertile men, 30-80\% have excessive production of reactive oxygen species (ROS) and decreased antioxidant capacity for various reasons, which ultimately lead to male infertility [1, 2].

Generally, oxidative stress (OS) is defined as an imbalance between the cell antioxidant defense system and the production of ROS [3]. OS is theorized to be one of the prominent biological molecular mechanisms of aging. Oxidative damage to the reproductive system is quite similar to male reproductive aging, and germ cells are particularly sensitive to OS with increasing age [4-7]. Thus, the disorder of the antioxidant system leads to a significant decline in the quality of germ cells with aging.

Previous studies have revealed that oral antioxidant treatment can remarkably improve sperm quality, elevate the live birth rate from $5 \%$ to $10-30 \%$, and enhance the clinical pregnancy rate from $6 \%$ to $11-28 \%$. In addition, antioxidant treatment has been established as the consensus treatment among international experts on male infertility $[8,9]$. In early studies, animal models such as aging, obesity, D-gal, triptolide, cyclophosphamide, and radiation models were widely used to assess antioxidant treatment methods [10-13]. For example, an aging mouse model indicated that melatonin supplementation could reduce the occurrence of abnormal spindle morphology and oocyte aneuploidy [14]. Moreover, in recent years, many mouse models with genetic defects associated with reproductive dysfunction phenotypes have been used in the field of reproductive medicine [1517]. The use of genetically deficient mice as model animals has become a clinical trend $[18,19]$. For instance, a Bmi1-KO mouse model has been used to prove that Bmi1 deletion can activate P16/P19 signaling and promote OS damage, ultimately leading to reproductive dysfunction. Supplementation with the antioxidant $\mathrm{N}$-acetyl-L-cysteine (NAC) can ameliorate the above outcomes [20]. All of these findings suggest that mice with antioxidation-related gene defects and reduced fertility can be used to evaluate the efficacy of antioxidants and to screen and study the effects of certain age-related antioxidants.

Traditional Chinese medicines (TCMs) can remarkably ameliorate male infertility [21]. Among them, Epimedium, Cuscuta, and Rhodiola have been used in the treatment of infertility for more than 2000 years and are extensively distributed in Asia, Europe, and other areas of the world [22-24]. Most related studies have

\footnotetext{
${ }^{1}$ Department of Histology and Embryology, Nanjing Medical University, Nanjing, China. ${ }^{2}$ Experimental Teaching Center of Basic Medicine, Nanjing Medical University, Nanjing,

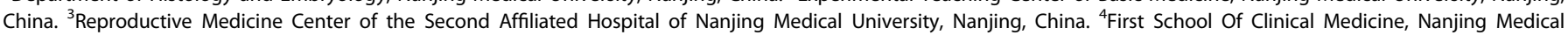
University, Nanjing, China. ${ }^{5}$ State Key Laboratory of Reproductive Medicine, Nanjing, China. ${ }^{6}$ These authors contributed equally: Haiyang Zhao, Tingting Zhao.

落mail: anniewang@njmu.edu.cn; qianyun@njmu.edu.cn

Edited by Professor Anastasis Stephanou
}

Received: 22 August 2021 Revised: 9 November 2021 Accepted: 19 November 2021

Published online: 20 January 2022 
focused on their pharmacological effects, such as their ameliorative effects on cardiovascular disease, osteoporosis, nervous system function, and aging [25-27]. In male reproduction, these TCMs can regulate reproductive endocrine function, promote sexual function, ameliorate spermatogenesis dysfunction, elevate sperm quality, adjust the hypothalamic-pituitary-testicular axis, improve erectile function, and exert other effects. The underlying mechanisms generally involve ROS and aging-related DNA damage [28-30]. However, it is not clear whether the specific mechanisms involve amelioration of OS-induced spermatogenesis in males via exertion of antioxidant effects and depletion of DNA damage.

Peroxisomal biogenesis factor 3 (PEX3) is a key protein in peroxisomal membrane synthesis that is present in all germ cells of the germinal epithelium [31] and can affect male reproductive function by regulating OS levels. One of the main functions of peroxisomes is to degrade ROS and thereby prevent cells from producing too much ROS through OS [32]. Peroxidase widely exists in spermatogenic cells of the testes as well as in testicular Sertoli-Leydig cells [33]. We found that Pex3-/- mice had abnormal sperm, male infertility, and increased testicular ROS levels but that the overall functioning of their other organs was completely normal. This finding indicated that Pex3-/- mice may be a good model for research on male reproductive function and the mechanisms of antioxidants. Therefore, in this study, Pex3-/mice were used as a model to study whether TCMs such as Epimedium [YYH], Cuscuta [TSZ], and Rhodiola [HJT] could ameliorate spermatogenesis dysfunction in mice. In further mechanistic studies, we deleted P16 (INK4a), a key gene in the age-related $\mathrm{P} 16-\mathrm{Rb}$ signaling pathway [34-36], in mice and constructed multigene deletion mice to further explore the specific mechanism of $\mathrm{YYH}$ in the amelioration of male agingrelated reproductive dysfunction.

\section{MATERIALS AND METHODS}

\section{Experimental design}

Three types of TCM granules (Tianjiang Pharmaceutical Co., Ltd., China) were selected in this study. The doses of administration were based on the conversion of the clinical doses of the drugs from humans to mice [37].

\section{Animals}

All mice were raised in a specific pathogen-free environment. All animal protocols were approved by the Animal Care and Use Committee of Nanjing Medical University. Pex3 and P16-KO mice were gifts from the research teams of Sha Jiahao and Miao Dengshun from the State Key Laboratory of Reproductive Medicine, Nanjing Medical University. All the animals were divided randomly into groups with no $<10$ mice in each group, as shown in Figure Legends. The mice were fed to the 9th week after birth, and then were administered continuously for 45 days by intragastric administration. During this period, we observed the mice every day, and directly eliminated the mice with the abnormal physiological states. The breeding methods for the mice with complex gene deletion are detailed in Figure S1. DNA was extracted from the tails of progeny mice, and the genes were amplified with specific primers. The genotypes of the mice were identified by DNA sequence analysis.

The primer sequences used were as follows: Pex3 primer 1: 5'-GCCAAACCATAGCACCAGC-3' Pex3 primer 2: $5^{\prime}$-CTTTGTCCTCTTTCTGGGCAC-3' Pex3 primer 3: 5'-TCGTGGTATCGTTATGCGCC-3' P16 primer 1: 5'- GGCAAATAGCGCCACCTAT-3' P16 primer 2: 5'-GCCGCTGGACCTAATAACTTC-3' P16 primer 3: 5'-GACTCCATGCTGCTCCAGAT-3'
A

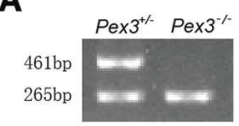

C
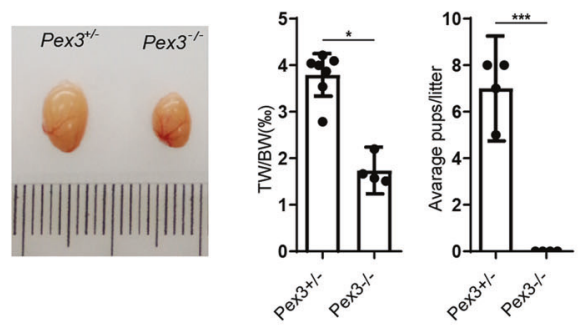

D

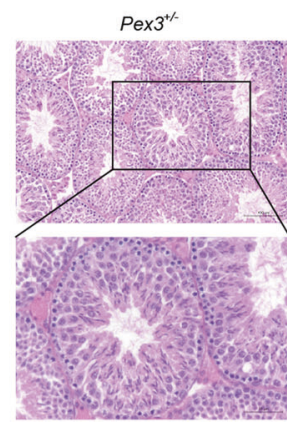

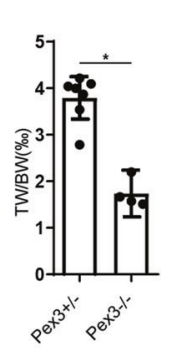
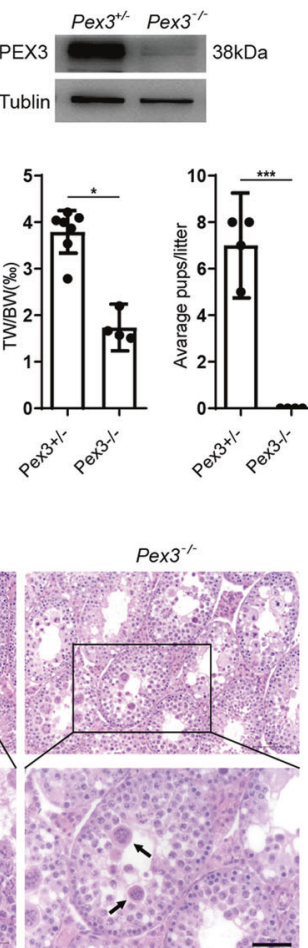

B
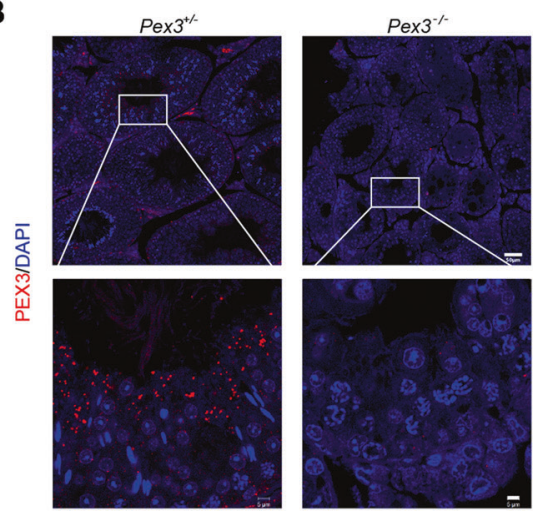

E

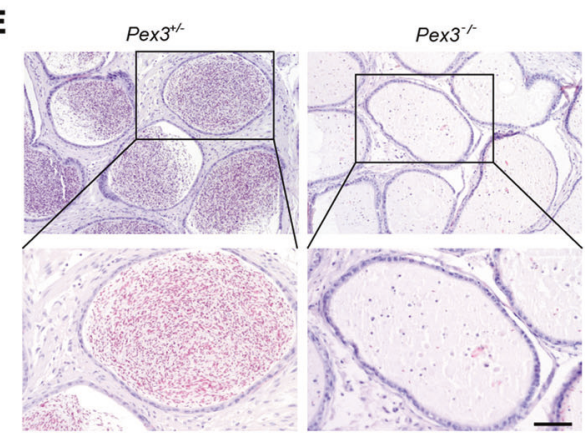

Fig. 1 Phenotypic validation of Pex3-KO mice. $(n=4-7)$. A Mouse tail DNA agarose gel electrophoresis and testicular protein immunoblotting; tubulin was used as an internal control. B PEX3 immunofluorescence (red) staining of testicular tissue. Scale bars: $5 \mu$ m. C Representative testicular morphology, testicular weight ratio (TW/BW), and natural reproduction results. D-E HE staining of the testis and epididymis. Scale bars: $50 \mu \mathrm{m}$. 
A

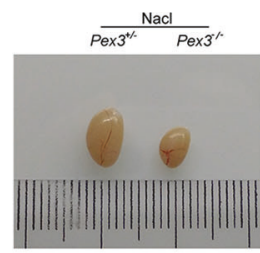

C
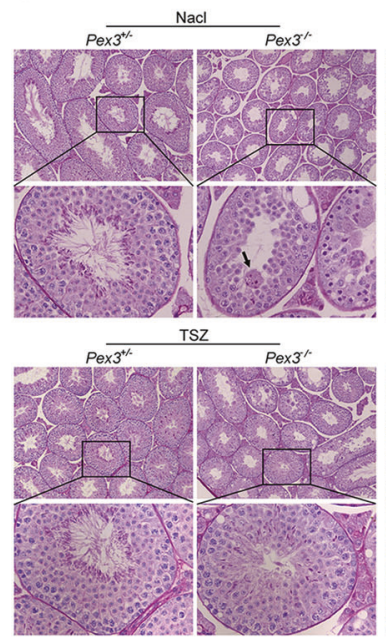

E

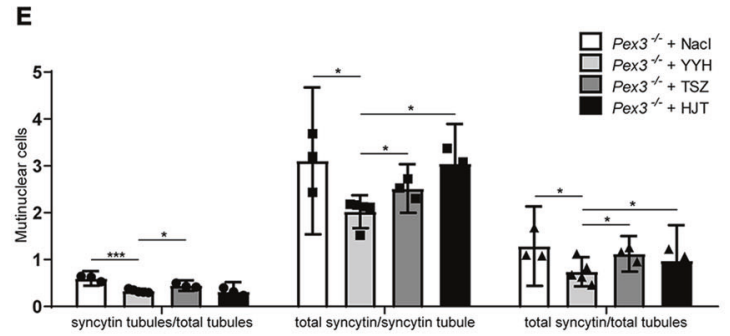

B

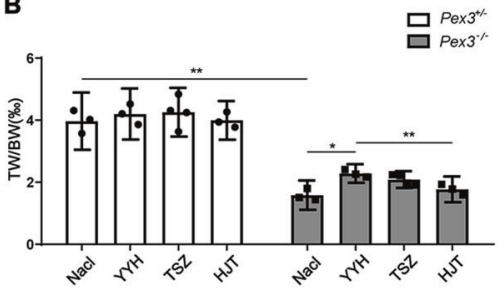

D
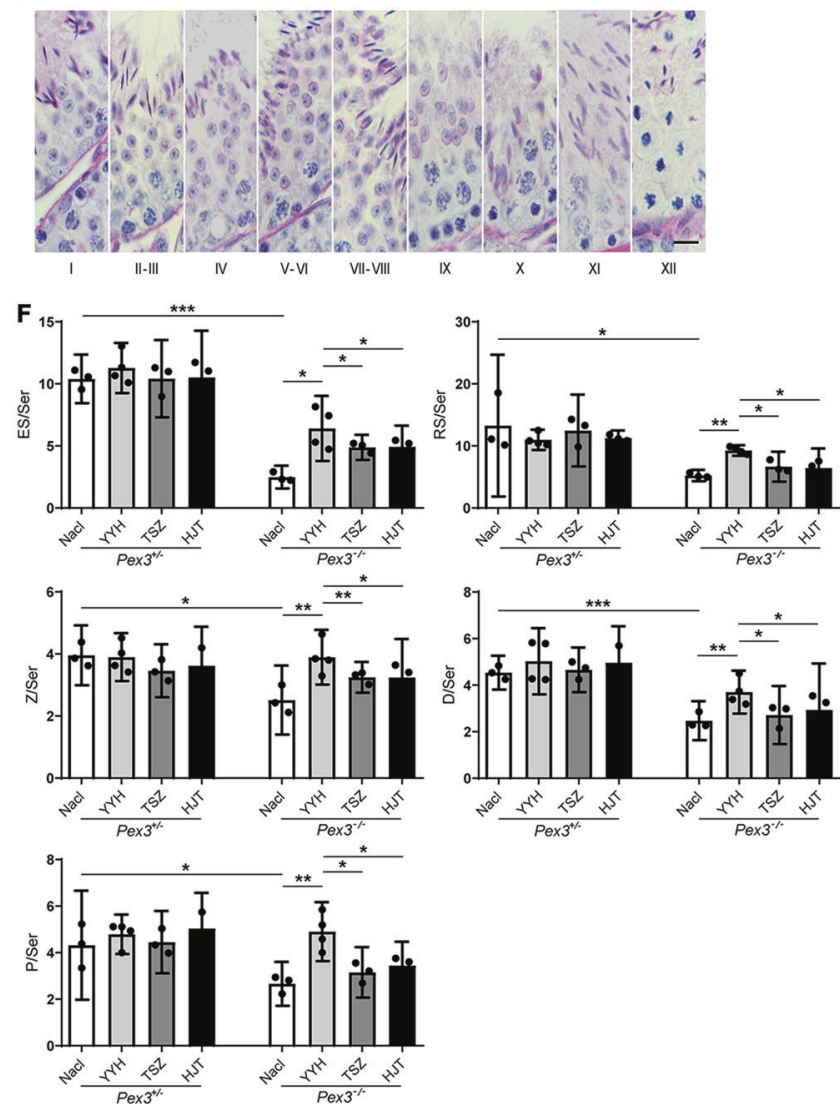

Fig. 2 Morphological comparison of testes treated with three different TCMs. $(\boldsymbol{n}=\mathbf{3 - 4})$. A Representative image of testicular sizes. B Testicular weight ratios. C HE staining of testes. Scale bars: $50 \mu \mathrm{m}$. D Criteria for distinguishing the time and phase of cells in the testicular lumen. Scale bars: $20 \mu \mathrm{m}$. E Statistics regarding syncytial multinucleated cells in the testicular lumen. $\mathbf{F}$ Sperm count statistics of each period. $E S$ elongated spermatid, $R S$ round spermatid, $Z$ zygotene spermatocyte, $D$ diplotene spermatocyte, $P$ pachytene spermatocyte.

\section{Body weight and reproductive organ weight}

The body weights of all of the experimental mice were measured weekly until the end of the experiment. The organ coefficients were calculated with the equation [(organ weight/body weight) $\times 100 \%$.

\section{Biochemical analysis of antioxidant status}

Frozen testis tissue was homogenized in ice-cold saline (testicular tissue weight:saline weight $=1: 9)$ for 20 minutes to prepare a $10 \%(w / v)$ homogenate. Then, the homogenates were filtered and centrifuged at $3500 \mathrm{rpm}$ and $4^{\circ} \mathrm{C}$ for 15 minutes using a refrigerated centrifuge, and superoxide dismutase (SOD) (S010M, Beyotime, China) and glutathione peroxidase (GSH-Px) (S0053, Beyotime) activity and malondialdehyde (MDA) (S0131, Beyotime) levels in the supernatant were determined using a microplate reader. The assay results were normalized to the protein concentration in each sample (P0010, Beyotime) and are expressed as $U$ $\mathrm{mg}^{-1}$ protein or $\mathrm{nmol} \mathrm{mg}^{-1}$ protein.

\section{ROS and apoptosis analysis}

Frozen sections of testicles were stained with a dihydroethidium (DHE) kit (R001, Vigorous Biol, China) according to the manufacturer's instructions. Paraffin sections of testes were stained with a TUNEL staining kit (A113, Vazyme, China) according to the manufacturer's instructions and then photographed with an LSM700 confocal microscope (Zeiss, Germany).

\section{Assessment of sperm motility and sperm count}

For animal sperm preparation, the caudal epididymis from adult mice was dissected and cut. Then, the tissues were incubated in human tubal fluid (HTF) medium (EasyCheck, China) at $37^{\circ} \mathrm{C}$. The sperm samples were diluted, and a $10 \mu \mathrm{L}$ aliquot of each sperm sample was evenly distributed on a glass chamber slide and analyzed using Computer Assisted Sperm Analyzer via the IVOS II $^{\mathrm{TM}}$ system (Hamilton Thorne, Beverly, MA, USA).

\section{Analysis of the sperm malformation rate}

To analyze the sperm malformation rate, $10 \mu \mathrm{L}$ of fresh spermatozoa were placed on slides. The air-dried slides were fixed in $4 \%$ paraformaldehyde and stained with hematoxylin-eosin (HE). Fifteen microscopic fields were randomly selected. Then, all sperm were counted, and the total number of sperm in each mouse was calculated. A count of at least 200 sperm was required. Finally, the abnormal sperm were carefully examined, and the proportion of sperm with abnormalities in the head and tail was calculated (by two technicians). 
A

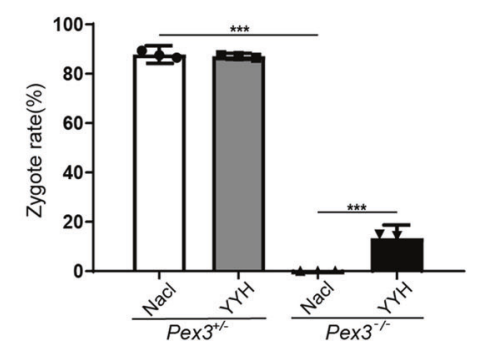

C

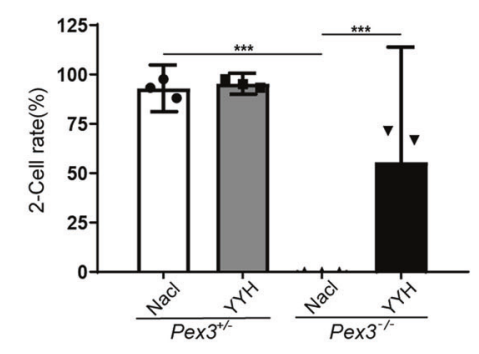

D

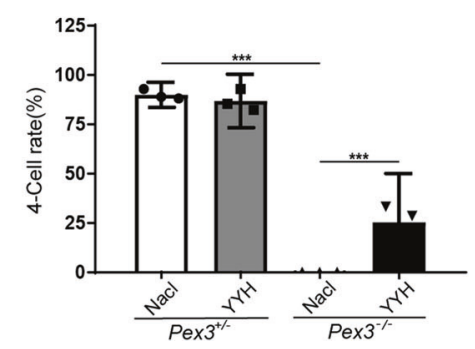

B
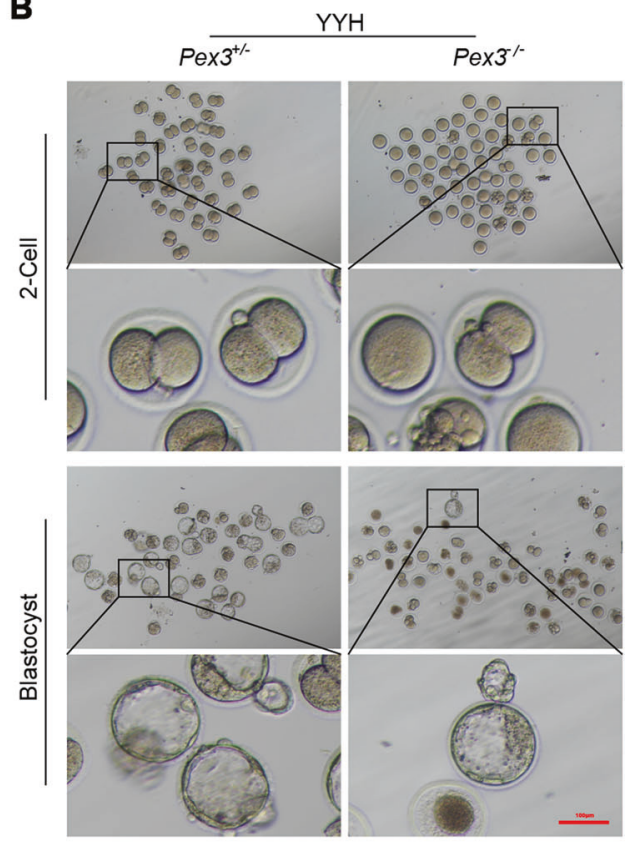

$\mathbf{E}$

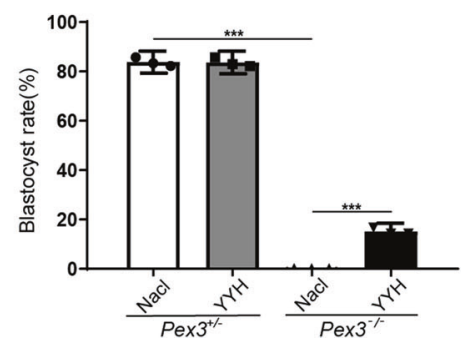

Fig. 3 Effects of Epimedium granule supplementation on fertility outcomes and early embryonic development of Pex3-KO mice ( $n=3$ ). A Fertilization rate. B Representative contrast images of the Pex3+/- and Pex3-/-groups in the IVF experiment and blastocyst embryos in male mice after YYH administration. Scale bars: $100 \mu \mathrm{m}$. C Two-cell embryo rate. D Four-cell embryo rate. E Blastocyst rate.

\section{Histological analysis}

Testes and epididymides were fixed overnight in Bouin solution (SLBJ3855V, Sigma). Then, the testes were dehydrated with an ethanol gradient. The testes were embedded in paraffin and cut into 5 - $\mu \mathrm{m}$-thick sections. The testicular sections were then dewaxed, rehydrated, and stained with HE (C0105s, Beyotime) and periodic acid-Schiff (PAS, 87007, Thermo). The seminiferous epithelium cycle stage of the testicular sections was determined according to morphological criteria. The staging was based primarily on the form and shape of the acrosome and to a lesser extent on the shape of the head of the sperm cell and the degree of chromatin condensation $[38,39]$.

\section{Immunohistochemistry and immunofluorescence analysis}

For immunofluorescence staining, prepared mouse tissue sections were treated at $4{ }^{\circ} \mathrm{C}$ with anti-PEX3 antibodies (121215, Abcam; 1:200); later, the sections were incubated with a 488-conjugated secondary antibody (SA00013-2, Proteintech; 1:500). An LSM700 confocal microscope (Zeiss) was used for imaging.

For immunohistochemical staining, tissue sections were treated at $4{ }^{\circ} \mathrm{C}$ with anti- $\gamma-H 2 A . x$ antibodies (9718T, CST; 1:200). Then, the samples were incubated with the secondary antibody and colorized with DAB (DA1015, Solarbio, China). Immunohistochemical analysis was performed under a brightfield microscope (Zeiss Axioskop 2 Plus).

\section{Western blotting}

Proteins were extracted with $8 \mathrm{M}$ urea lysis buffer $(8 \mathrm{M}$ urea, $75 \mu \mathrm{M} \mathrm{NaCl}$, $50 \mu \mathrm{M}$ Tris-Cl pH 8.2) containing $1 \mathrm{mM}$ phenylmethylsulfonyl fluoride and quantified using a Bradford Protein Assay Kit (Beyotime). The protein samples were separated in gradient gel and transferred to PVDF membranes (Millipore). The antibodies were as follows: anti-PEX3 (A7352, Abclonal; 1:1000), anti-P16 (211542, Abcam; 1:1500), anti-CDK2 (2546 S, CST; 1:1000), anti-CDK6 (3136 S, CST; 1:2000), anti-P19Arf (10272, Proteintech; 1:2000), anti-P53 (2524 T, CST; 1:1000), anti-GAPDH (60004, Proteintech; 1:2000), and anti- $\beta$-Actin (66009, Proteintech; 1:2000). The samples were incubated with the appropriate primary antibodies overnight at $4{ }^{\circ} \mathrm{C}$ after blocking in $5 \%$ nonfat milk. After washing three times in TBST, the samples were incubated with secondary antibodies at the appropriate dilutions for $2 \mathrm{~h}$ at room temperature. The results were recorded after enhanced chemiluminescence color development (Tanon, China). In this study, we used Image J software to calculate the gray value, and the ratio of the gray value of the internal reference protein in the same lane is regarded as the final result.

\section{In vitro fertilization (IVF) assay}

Female C57B6 mice (6-8 weeks old) were injected with pregnant mare serum gonadotropin (5 IU, Ningbo Sansheng Pharmaceutical Co., Ltd., China) $48 \mathrm{~h}$ before human chorionic gonadotropin (HCG) (5 IU, Ningbo Sansheng Pharmaceutical Co., Ltd., China) treatment. After $16 \mathrm{~h}$ of stimulation with $\mathrm{HCG}$, oocytes were collected in pre-equilibrated oocyte IVF medium. The oocytes in HTF medium (Easy Check) were mixed with the activated epididymal sperm, incubated at $37^{\circ} \mathrm{C}$ at $5 \% \mathrm{CO}_{2}$, placed in $\mathrm{KSOM}$ medium (Easy Check), and cultured undisturbed for 4 days. The developing embryos were identified microscopically based on progression to the blastocyst stage $(n=200)$. 
A

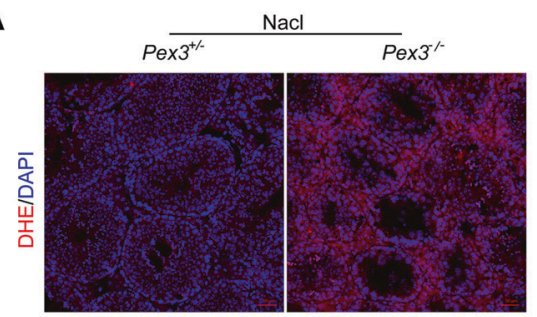

B

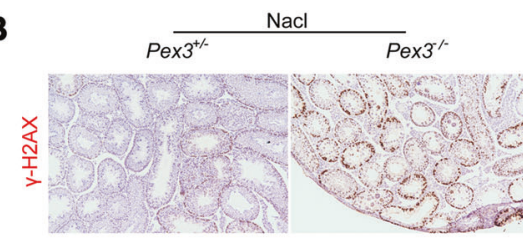

C

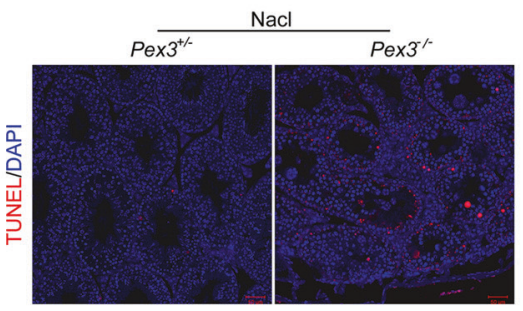

D

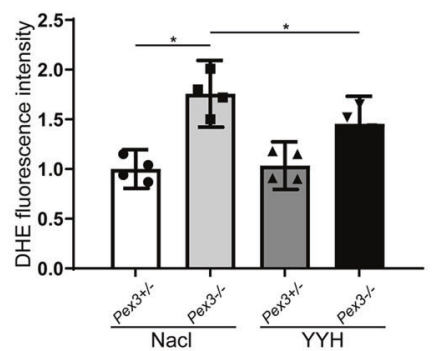

F

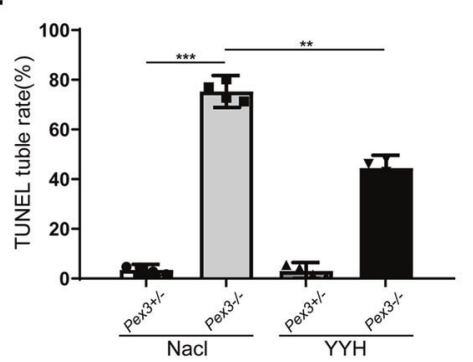

H

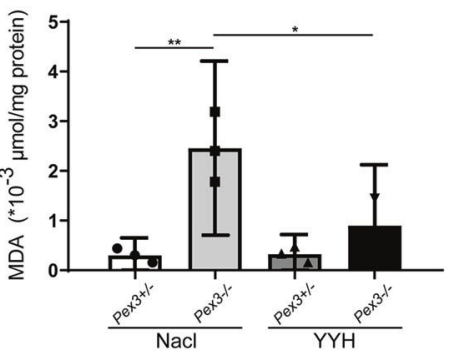

YYH

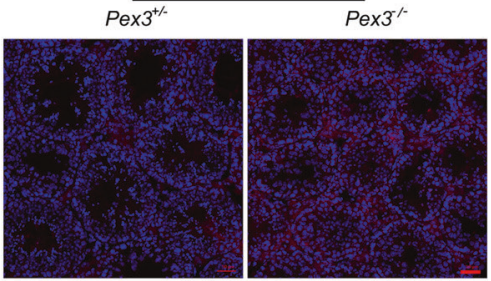

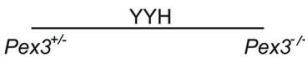
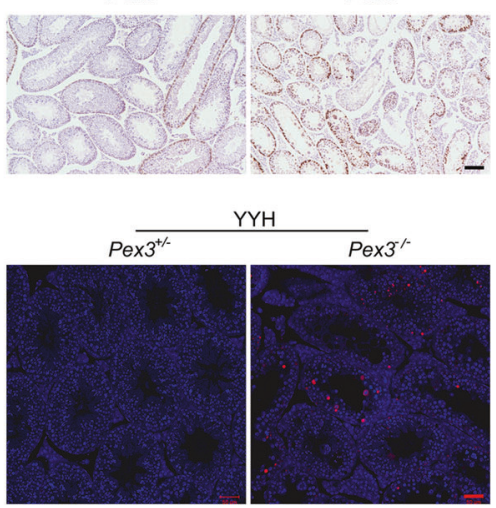

E

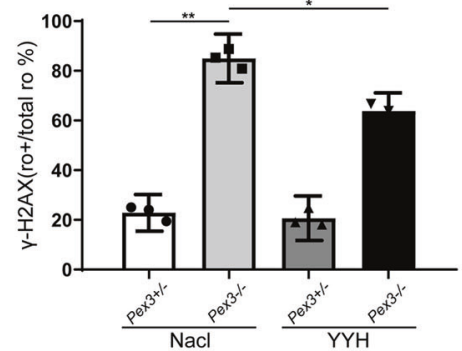

G

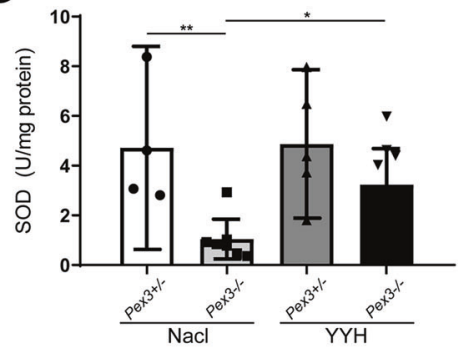

I

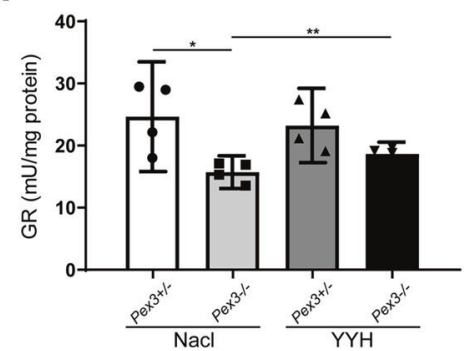

Fig. 4 Effects of YYH supplementation on OS levels in testicular tissues of Pex3-KO mice. $(\boldsymbol{n}=\mathbf{3 - 8})$. A DHE fluorescence (red) in testicular tissue from each group. Scale bars: $50 \mu \mathrm{m}$. B Images of $\gamma$-H2A.X immunohistochemical staining in testicular tissue from each group. Scale bars: $100 \mu \mathrm{m}$. C Image of TUNEL staining (red fluorescence) showing testicular apoptosis in each group. Scale bars: $50 \mu \mathrm{m}$. D DHE fluorescence intensity. E Rate of $\gamma$-H2AX-positive luminal cells. F Rate of TUNEL-positive luminal cells. G SOD level. H MDA level. I GR level. 
A

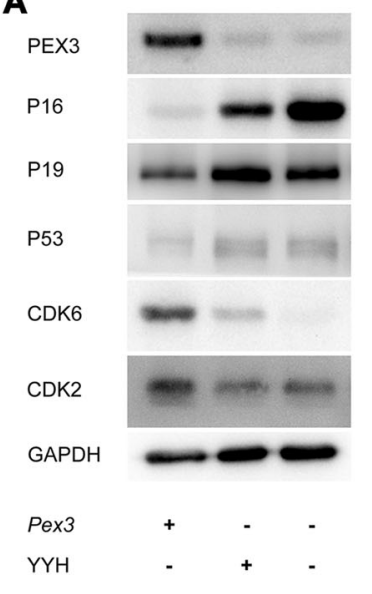

B

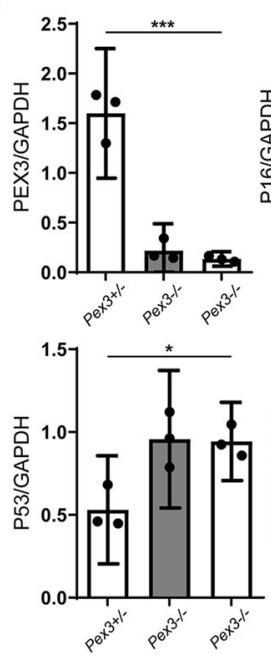

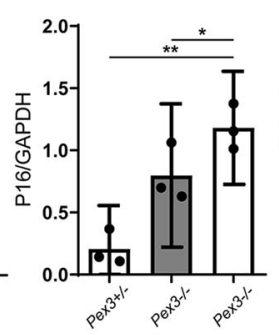
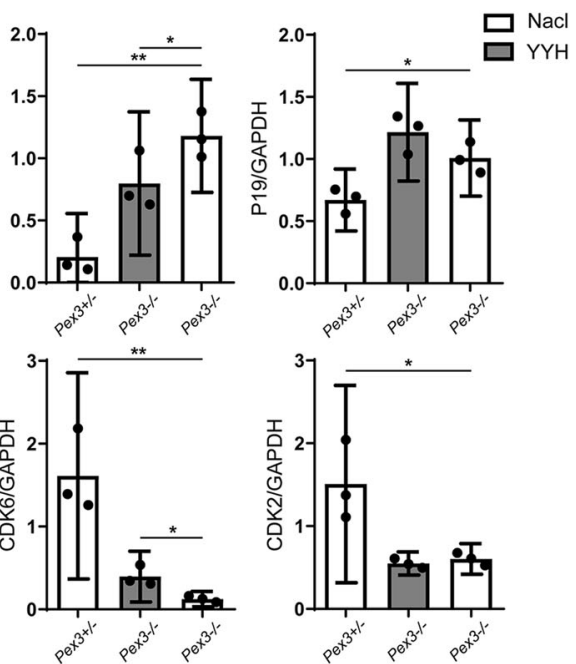

Fig. 5 Detection of the expression levels of senescence pathway-related proteins after YYH supplementation. ( $n=3$ ). A Western blot results for P16-Rb and P53-P19 signaling-related proteins. B Grayscale values.

Table. 1. Design of the experiment.

\begin{tabular}{|c|c|c|c|}
\hline Group & Genotype & Treat & Dose \\
\hline 1 & Pex3+I- & $\mathrm{Nacl}$ & $300 \mu \mathrm{L}$ \\
\hline II & Pex3-/- & $\mathrm{Nacl}$ & $300 \mu \mathrm{L}$ \\
\hline III & Pex3+I- & YYH & $325 \mathrm{mg} / \mathrm{Kg} /$ day \\
\hline IV & Pex3-/- & YYH & $325 \mathrm{mg} / \mathrm{Kg} /$ day \\
\hline V & Pex3+I- & TSZ & $325 \mathrm{mg} / \mathrm{Kg} /$ day \\
\hline VI & Pex3-/- & TSZ & $325 \mathrm{mg} / \mathrm{Kg} /$ day \\
\hline VII & Pex3+I- & HJT & $487 \mathrm{mg} / \mathrm{Kg} /$ day \\
\hline VIII & Pex3-/- & HJT & $487 \mathrm{mg} / \mathrm{Kg} /$ day \\
\hline IX & Pex3+I-P16+I- & $\mathrm{Nacl}$ & $300 \mu \mathrm{L}$ \\
\hline $\mathrm{x}$ & Pex3+I-P16+I- & YYH & $325 \mathrm{mg} / \mathrm{Kg} /$ day \\
\hline$X I$ & Pex3-/-P16+I- & $\mathrm{Nacl}$ & $300 \mu \mathrm{L}$ \\
\hline XII & Pex3-/-P16+I- & YYH & $325 \mathrm{mg} / \mathrm{Kg} /$ day \\
\hline XIII & Pex3-/-P16-/- & $\mathrm{Nacl}$ & $300 \mu \mathrm{L}$ \\
\hline XIV & Pex3-/-P16-/- & YYH & $325 \mathrm{mg} / \mathrm{Kg} /$ day \\
\hline$x V$ & Pex3+I-P16-/- & $\mathrm{NaCl}$ & $300 \mu \mathrm{L}$ \\
\hline $\mathrm{XVI}$ & Pex3+I-P16-/- & YYH & $325 \mathrm{mg} / \mathrm{Kg} /$ day \\
\hline
\end{tabular}

\section{Assessment of fertility}

To determine fertility, a male mouse was arranged to mate with two female mice. After normal vaginal plug formation was detected, the female mice were separated from the male mouse. The number of offspring produced by each female was recorded.

\section{Statistical analysis}

All data analysis was carried out without distinguishing between experimental groups. All assays were repeated at least three times. SPSS 19.0, Excel, and GraphPad Prism 8.0. were used for statistical analysis. The statistical significance of differences in mean values was assessed with $t$ tests $\left({ }^{*} P<0.05\right.$; $\left.{ }^{* *} P<0.01 ;{ }^{* *} P<0.001\right)$. Data points correspond to the mean of the independent experiments and error bars denote standard deviation.

\section{RESULTS}

Verification and preliminary evaluation of the reproductive function of Pex3-KO mice

We eventually confirmed the successful construction of Pex3-KO mice (Fig. 1A/B), and the results of several phenotypic experiments showed that compared with wild-type male mice, Pex3-KO male mice had abnormal testicular development (Fig. 1C) and reduced testicular volumes and organ ratios and were completely infertile. Moreover, the results of HE staining of Pex3-KO mice revealed an abnormal testicular lumen structure (Fig. 1D). Sperm were completely absent from the epididymis (Fig. 1E), indicating spermatogenesis blockade. Unique pathological structures called syncytia [40] were present in the testicular lumen in Pex3-KO mice (arrow), which further proved the arrest of spermatogenesis.

Different TCM supplements had different ameliorative effects on dyszoospermia in Pex3-KO mice, among which YYH had the best effect

To investigate how to improve spermatogenesis in Pex3-KO mice, we selected three TCMs (TSZ, HJT, and YYH) for rescue experiments. A preliminary comparison revealed that YYH had the best effect. Subsequently, comparisons of testicular morphologies, sperm structures, and testicular seminiferous epithelial cycles revealed several findings. First, treatment of Pex3-KO mice with TCMs increased the testicular weight ratio and attenuated the abnormal testicular development (Fig. 2A, B). It also ameliorated dyszoospermia by increasing the number of elongated spermatids (Fig. 2D, F) and reduced the number of syncytial multinucleated cells so that testicular degeneration was ameliorated (Fig. 2C, E). YYH was more effective than TSZ and HJT. For the wild-type male mice, there was no significant change in any index with TCM supplementation. The above results implied that the three TCMs all improved the spermatogenesis disorder caused by Pex3-KO to a certain extent and the rescue effect of YYH was significantly better than those of the other two TCMs.

\section{YYH supplementation enabled Pex3-KO mice to produce transplantable embryos}

After confirming that supplementation with YYH among the three TCMs significantly ameliorated spermatogenesis disorder in Pex3$\mathrm{KO}$ mice, we further explored whether YYH supplementation had a rescue effect on the reproductive outcome. Consistently, the results indicated that $\mathrm{YYH}$ administration did not remarkably facilitate the ability of Pex3-KO mice to undergo natural pregnancy, but it increased the potential for obtaining early embryos. Early embryos were successfully obtained through IVF in the YYH-treated Pex3-KO (YYH-Pex3-/-) group (Fig. 3B), and the developmental ability of embryos was notably improved (Fig. 3A, $\mathrm{C}-\mathrm{E})$. Male $\mathrm{NaCl}$-treated Pex3-KO (NaCl-Pex3-/-) mice could not undergo IVF due to the absence of sperm production. The above 
A

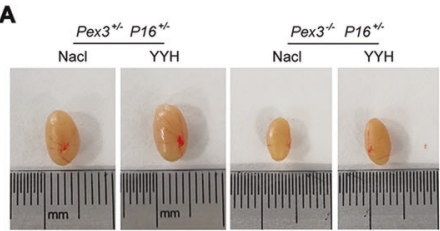

B

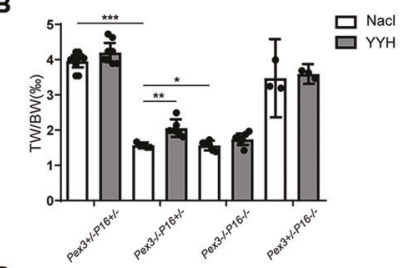

D

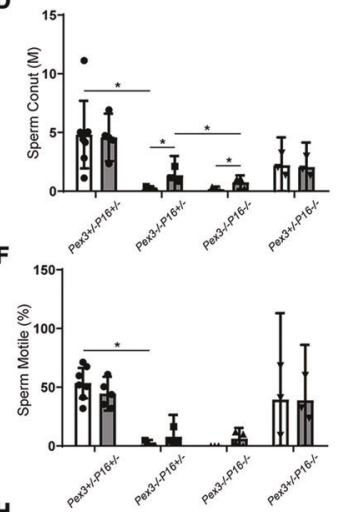

H

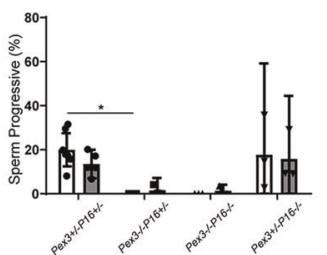

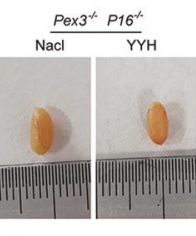

E
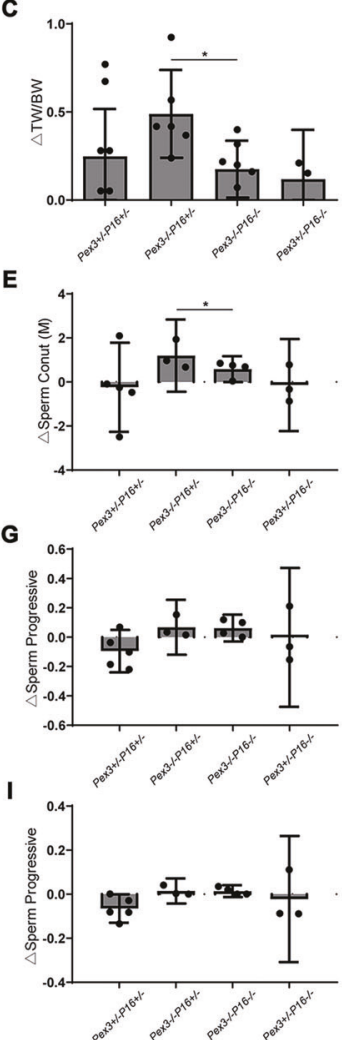
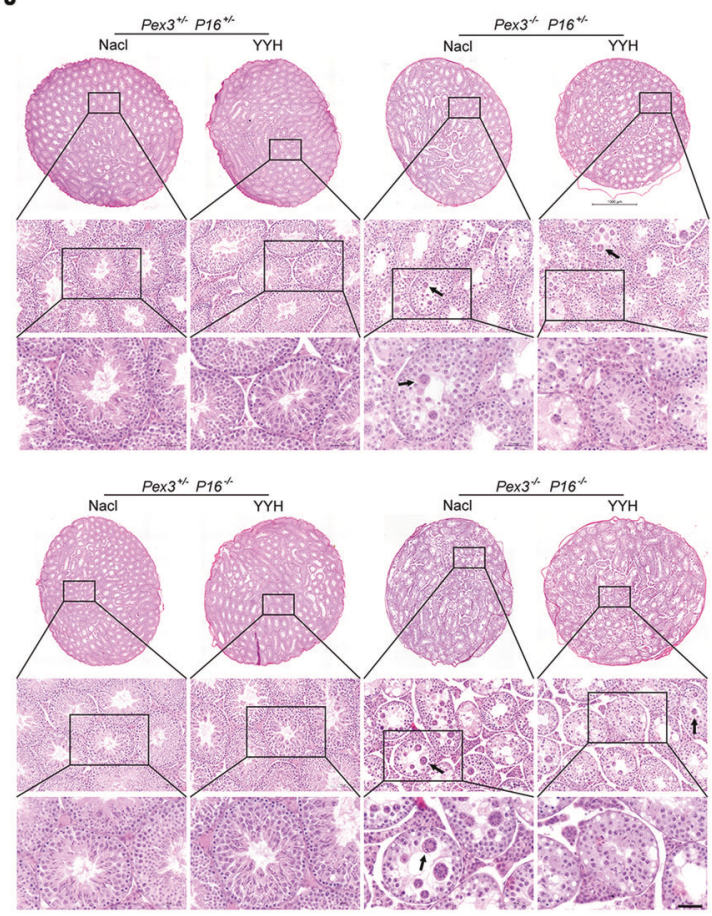

K
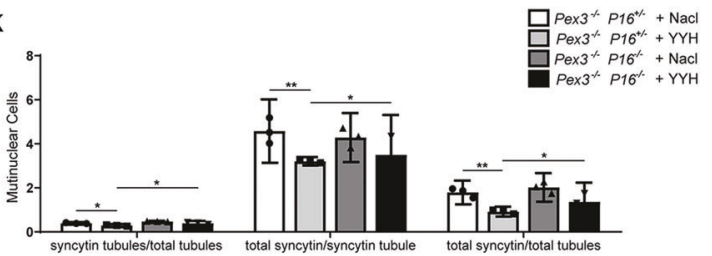

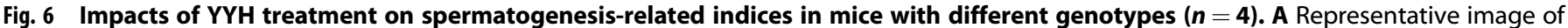
testicular sizes. B Testicular weight ratios. C Changes in the testicular weight ratio. D Sperm counts. E Changes in the sperm count. F Sperm motility. G Changes in sperm motility. H Sperm forward motility. I Changes in sperm forward motility. $\mathbf{J}$ Images of HE staining in testes. Scale bars: $50 \mu \mathrm{m}$. K Statistics regarding syncytial multinucleated cells in the testicular lumen.

results demonstrated that although YYH supplementation did not improve the natural reproductive outcomes of Pex3-KO male mice, it enabled early embryos to be obtained, which made reproduction possible.

\section{YYH supplementation effectively improved OS in the testes of Pex3-KO mice}

Given the strong antioxidant function of $\mathrm{YYH}$, we investigated whether $\mathrm{YYH}$ exerted a rescue effect by improving antioxidation in Pex3-KO mouse testes. We compared the levels of ROS (with the fluorescent probe DHE), DNA damage (via $\mathrm{yH} 2 \mathrm{~A} . \mathrm{X}$ detection), apoptosis (via TUNEL staining), lipid peroxidation (via MDA detection), SOD activity, and glutathione reductase in the testicular tissues of male mice. The results revealed that compared with the $\mathrm{NaCl}-\mathrm{Pex} 3-/-$ group, the $\mathrm{YYH}-\mathrm{Pex3}-/-$ group showed lower ROS levels (Fig. 4A, D); less DNA damage (Fig. 4B, E), apoptosis (Fig. 4C, F), and lipid peroxidation (Fig. 4H); and greater antioxidant capacity (Fig. 4G, I). However, the wildtype male mice displayed no apparent changes. The above results proved that YYH depleted OS injury by enhancing the antioxidant levels and reducing the ROS levels in spermatogenic cells. These changes reduced DNA oxidative damage and apoptosis in spermatogenic cells and ultimately enabled amelioration of spermatogenesis disorder and improvement of reproductive outcomes.

\begin{abstract}
YYH supplementation attenuated reproductive dysfunction in Pex3-KO mice by regulating the expression levels of proteins related to the cell senescence pathway

In this study, we found a series of testicular senescence-related phenotypes, such as increased testicular luminal cell apoptosis, DNA damage and ROS levels as well as spermatogenic cell degeneration. These findings implied that the senescence pathway was a good entry point to conduct in-depth studies on the mechanism of $\mathrm{YYH}[41,42]$. We, therefore, analyzed the protein expression levels of common genes related to the aging pathway and found that the deletion of the Pex3 led to upregulation of the protein expression levels of P19 and P16 and that P16 exerted an inhibitory effect to downregulate the expression level of the downstream protein CDK6 (Fig. 5A/B). In addition, the deletion of PEX3 contributed to the upregulation of the protein level of P53. In addition, P53 inhibited the downstream protein CDK2 to downregulate its expression level. The reductions in the expression levels of CDK2 and CDK6 led to activation (phosphorylation) of the downstream protein $\mathrm{Rb}[43,44]$, causing cell development arrest and apoptosis and ultimately resulting in spermatogenesis disorder. YYH supplementation ameliorated this damage phenotype to a certain extent. Similarly, we found that YYH supplementation attenuated the increase in $\mathrm{P} 16$ protein level caused by the deletion of the Pex3 gene, but it did not significantly affect the protein levels of P19 and P53 (Fig. 5A, B).
\end{abstract}


A
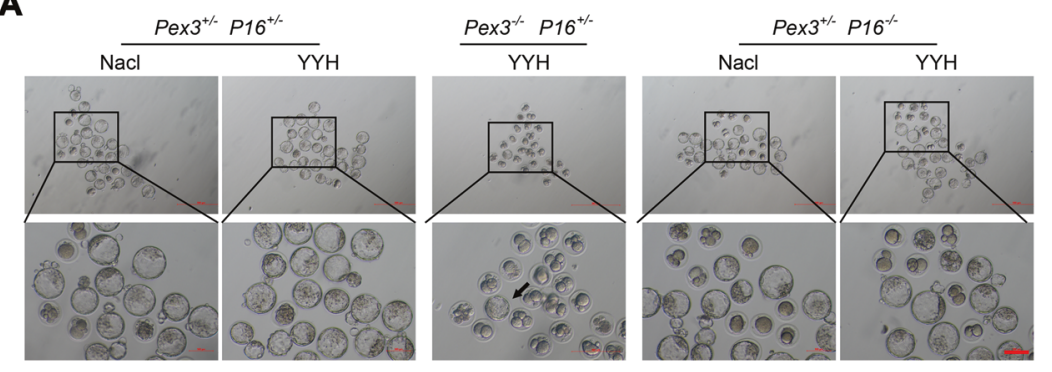

B

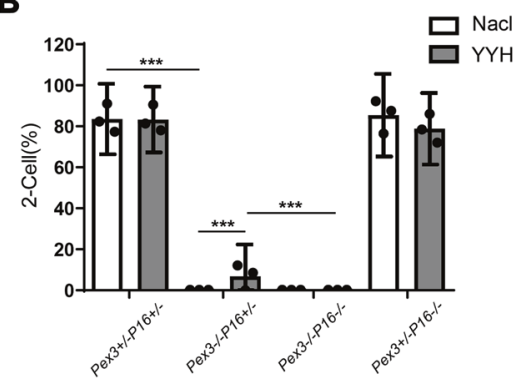

C

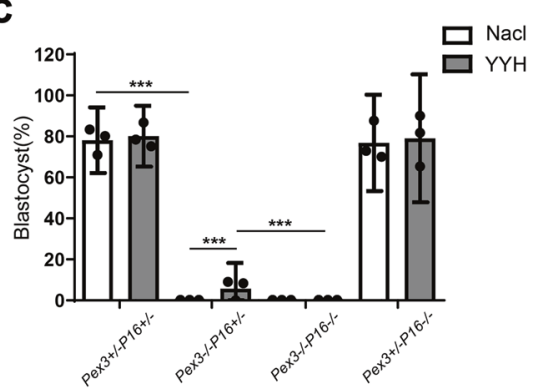

Fig. 7 Effects of YYH treatment on early embryonic development in mice with different genotypes. $(n=3)$. A Contrast images of blastocyst-stage embryos from each group. Scale bars: $100 \mu \mathrm{m}$. B Two-cell embryo rate. C Blastocyst rate.

YYH ameliorated the spermatogenesis dysfunction phenotype induced by Pex3-KO through a P16-dependent pathway As previously mentioned, YYH had a beneficial effect on spermatogenesis in Pex3-KO mice. In addition, it significantly inhibited the abnormal increase in the expression of P16 in the absence of PEX3. We consequently hypothesized that the P16CDK6-Rb signaling pathway mediated the effect of $\mathrm{YYH}$ on spermatogenesis in Pex3-KO mice. To test this hypothesis, we introduced multiple combinations of gene deletions (Fig. S1). YYH administration groups and normal saline administration groups were included in the experiments (Table 1). By analyzing testicular morphology, testicular weight, sperm counts/motility/progressive motility, and testicular microstructure, we found that YYH administration attenuated the testicular abnormalities caused by Pex3-KO (Pex3-/-P16+/-) and increased the testicular organ ratio. The effect was weakened when $P 16$ was knocked out (Pex3-/-P16-/-) (Fig. 6A, B, C). YYH administration also significantly increased the sperm counts of Pex3-/-P16+/- and Pex3-/-P16-/- mice (Fig. 6D, E), and the effect was better in the Pex3-/-P16+/- group. Additionally, YYH administration tended to improve the sperm quality of $P$ ex3 $-/-P 16+/-$ and Pex3-/-P16-/ - mice, but the results were not statistically significant (Fig. 6F-I). Furthermore, according to the testicular microstructure analysis, YYH administration markedly reduced the syncytial multinucleated cell abundance of Pex3-KO mice but had no significant effect on that of Pex3-/-P16-/- mice (Fig. 6J, K). Similarly, single deletion of the $P 16$ gene did not significantly affect spermatogenesis. In conclusion, the ability of $\mathrm{YYH}$ to ameliorate spermatogenesis dysfunction phenotypes of Pex3-KO mice was reduced when $P 16$ was knocked out.

\section{The beneficial effect of YYH on IVF outcomes in Pex3-KO mice vanished in the absence of $P 16$}

We further investigated more comprehensively whether P16-KO affected the reproductive outcomes of YYH-treated Pex3-KO mice. The results revealed that YYH-Pex3-/-P16-/- mice had no sperm production and could not undergo IVF. However, early embryos were again able to be successfully obtained from YYH-Pex3-/-P16+/- mice by IVF (Fig. 7A), and the proportions of embryos that reached the two-cell and blastocyst stages were also remarkably elevated (Fig. 7B, C). These results suggested that YYH improved the reproductive outcomes of Pex3-KO mice, thus enabling transplantable embryos to be obtained. However, the effect of YYH on reproductive outcomes disappeared when the P16 gene was knocked out.

\section{The beneficial effect of YYH on OS in Pex3-KO mice was attenuated in the absence of $P 16$}

We also hypothesized that $P 16-\mathrm{KO}$ affected the antioxidant activity of YYH in Pex3-KO mice. We compared the levels of OS-induced DNA damage and apoptosis in testicular tissues of the mice in each group and found that $\mathrm{YYH}$ administration ameliorated the peroxidation damage caused by $P$ ex 3 single-gene $\mathrm{KO}$, reduced the numbers of $\mathrm{\gamma H} 2 \mathrm{Ax}$-positive luminal cells (Fig. 8A, C, D) and apoptotic luminal cells (Fig. 8B, E), and decreased the levels of MDA (Fig. 8F) and SOD (Fig. 8G). YYH administration also improved these indices in Pex3-/ $P 16-/-$ mice, but the rescue effects on DNA damage and apoptosis were markedly weaker than those in Pex3-KO mice. The above results indicated that when P16 was knocked out, the ameliorative effect of YYH on OS in Pex3-KO mice was observably weakened. Hence, we conclude that $\mathrm{YYH}$ is able to exert antioxidant functions through a P16-dependent pathway to improve male infertility caused by Pex3-KO.

\section{DISCUSSION}

It is well known that OS is a prominent factor in male infertility or subfertility. Presently, antioxidant supplementation is one of the most common methods of male infertility treatment. TCMs with antioxidant functions, such as Epimedium, is increasingly being used to ameliorate male infertility caused by high levels of OS [45-50]. Nevertheless, due to the complexity of TCM components, most traditional pharmacological studies have focused mainly on the beneficial effect of a single component, such as flavonoids and icariin in $\mathrm{YYH}$. While in practical clinical applications, multicomponent TCMs are mostly used, the precise application of which needs to be further improved. The previous findings inspired us to investigate the efficacy and mechanism of clinical TCMs in order to provide more support for precision medicine. 
A

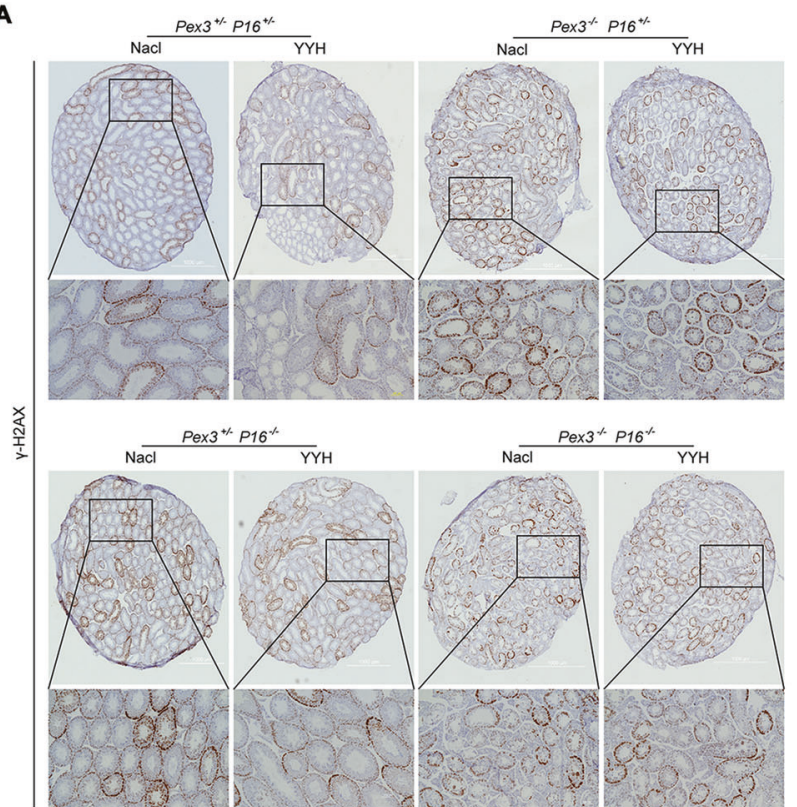

C
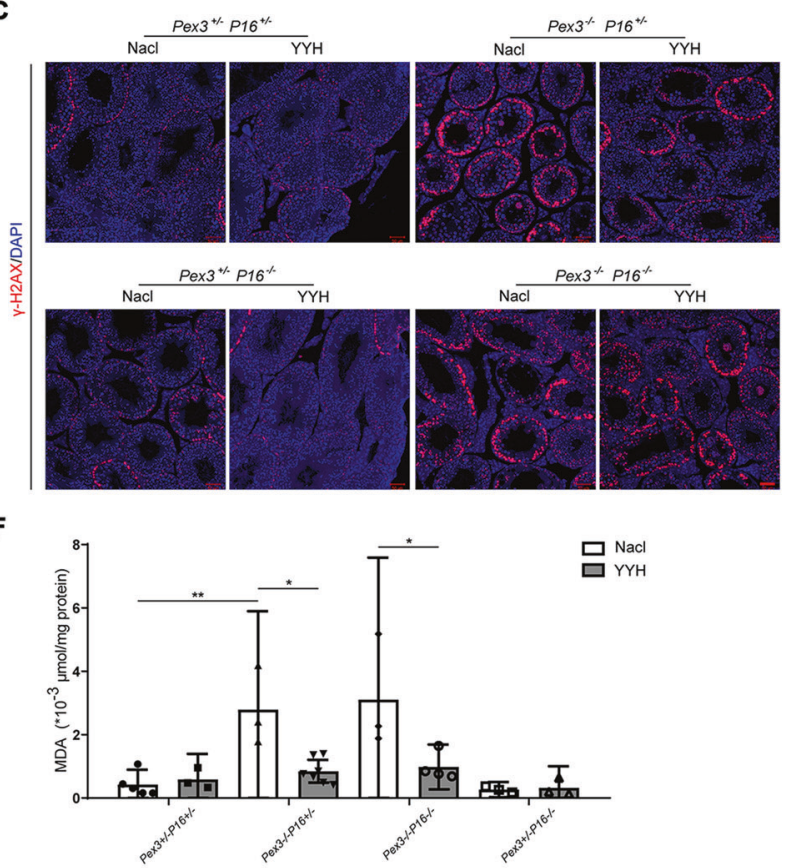

B

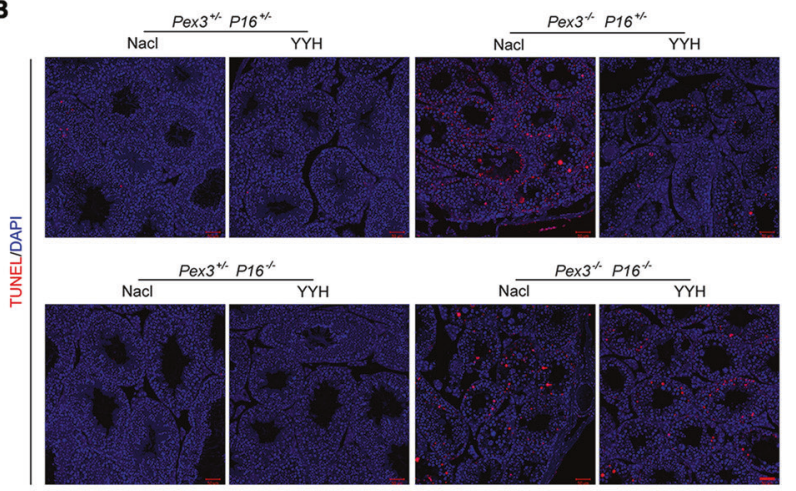

D

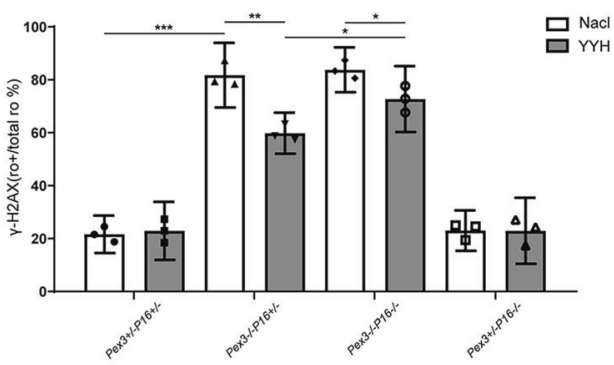

E
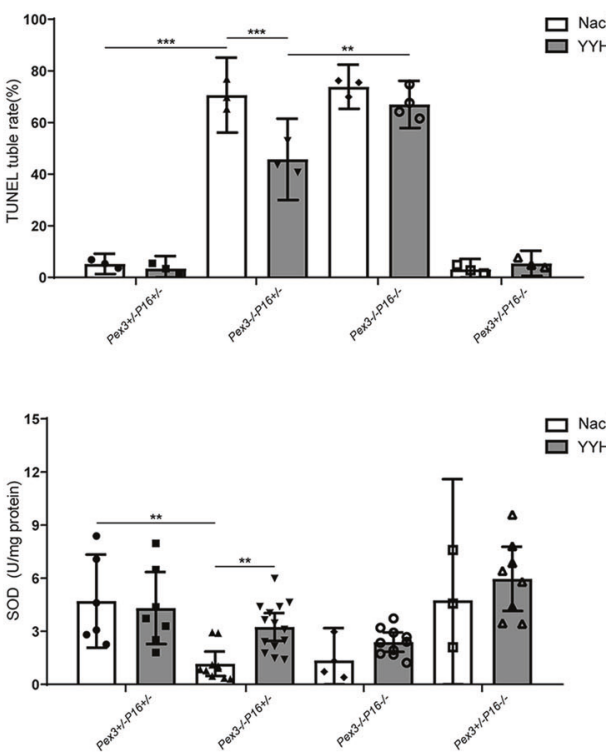

Fig. 8 Effects of YYH supplementation on OS levels in testicular tissues of mice with different genotypes. $(n=3-6)$. A $\gamma$ - $\mathrm{H} 2 \mathrm{AX}$ immunohistochemistry in testicular sections. Scale bars: $100 \mu \mathrm{m}$. B TUNEL staining of testicular sections. Scale bars: $50 \mu \mathrm{m}$. C $\gamma$-H2AX immunofluorescence in testicular sections. Scale bars: $50 \mu \mathrm{m}$. D Rate of $\gamma-\mathrm{H} 2 \mathrm{AX}$-positive luminal cells. E Rate of TUNEL-positive luminal cells. $\mathbf{F}$ MDA level. G SOD level.

To achieve this goal, we established mice with Pex3-KO. Preliminary studies revealed that Pex3-KO led to dyszoospermia and male infertility in male mice, which suggested that the Pex3KO mouse model was a good model with which to study the reproductive effects and mechanisms of antioxidants (Fig. 1). We combined this model with clinical practice to develop a novel approach. Instead of studying a single component, we directly studied the rescue effects of three novel Chinese herbal formula granules with antioxidant functions. The results revealed that $\mathrm{YYH}$ supplementation resulted in a small number of spermatids (Fig. 2), and the following IVF study showed that a few transplantable embryos could be obtained from Pex3-KO mice (Fig. 3). This result suggests that $\mathrm{YYH}$ supplementation may help improve the fertilization potential and the outcome of assisted reproductive technology (ART). More importantly, during in vitro ART treatment, we found that poorer embryo quality was associated with a greater negative paternal contribution. Normally, the relative influence of sperm on the ART outcome is $10-15 \%$. However, an abnormal paternal component strongly affects fertility and the outcome of ART. We, therefore, hypothesize that YYH can be used as the preferred TCM for male infertility with elevated OS to increase the potential of success for male ART patients with severe spermatogenesis disorders [51-53].

In addition, we found increased ROS levels, decreased antioxidant capacity, and elevated DNA damage levels in Pex3$\mathrm{KO}$ mice (Fig. 4). Subsequently, a series of age-related phenotypes 
emerged $[54,55]$. We also confirmed that the expression level of P16 and its downstream CDK6 protein had a decreasing tendency (Fig. 5). All of the above results inspired us to study the aging pathway as the breakthrough point [56-58]. YYH supplementation remarkably promoted the phenomenon mentioned above, implying that YYH can indeed improve reproductive function through the ROS-DNA damage-P16-CDK6 signaling pathway. Subsequently, we found that supplementation with $\mathrm{YYH}$ did not exert a significant positive effect on young Pex3-/-P16-/- mice, which indicated that inhibition of the senescence pathway mediated mainly by P16 affected YYH supplementation to some extent even under OS.

Although it would have been common and convenient to further explore YYH's rescue effect on spermatogenetic blockade related to P16 and the cell senescence pathway using cell lines as models, such a design would not have reflected the real in vivo situation and would have had limited reference value for clinical precision medicine. Hence, we established Pex3 and P16 multigene-KO mice (Fig. S1). We found that KO of $P 16$ weakened the rescue effect of YYH on testicular dysplasia and dyszoospermia caused by Pex3-KO (Fig. 6). Moreover, there were no significant rescue effects on DNA damage and apoptosis, and antioxidant activity was weakened (Fig. 8). Similarly, in Pex3-KO mice with concurrent $P 16-\mathrm{KO}$, transplantable embryos could not be obtained after $\mathrm{YYH}$ supplementation (Fig. 7). In general, when the $P 16$ was knocked out, the rescue effect of $\mathrm{YYH}$ on reproductive dysfunction caused by Pex3-KO was weakened. We speculate that the significant P16-KO-induced weakening of the rescue effect of YYH occurred because P16 is a key target of YYH. Additionally, we observed that P16 expression is age-dependent $[59,60]$, which suggests that although $\mathrm{YYH}$ is the preferred TCM for male infertility with increased $O S$, the effect in young people may not be adequate.

In conclusion, our findings suggest that YYH can reduce ROS levels and DNA damage through antioxidant activity. In addition, $\mathrm{YYH}$ regulates the level of P16 protein expression, attenuating excessive P16 protein expression and thereby regulating downstream CDK6 levels, promoting cell proliferation, inhibiting apoptosis, and decreasing abnormal spermatogenic cell arrest. The results show that by mediating ROS-DNA damage-P16-CDK6 pathway signaling, $\mathrm{YYH}$ exerts antioxidative effects and can ameliorate clinical male infertility caused by $O S$ and enhance the contributions of male factors in ART. In the future, combining antioxidant supplementation with YYH as the core ingredient with modern ART will open up endless possibilities for male patients with severe infertility.

\section{DATA AVAILABILITY}

The data that support the findings of this study are available from the corresponding author upon reasonable request.

\section{REFERENCES}

1. Tremellen K. Oxidative stress and male infertility-a clinical perspective. Hum Reprod Update. 2008;14:243-58.

2. Smits RM, Mackenzie-Proctor R, Yazdani A, Stankiewicz MT, Jordan V, Showell MG. Antioxidants for male subfertility. Cochrane Database Syst Rev. 2019;3:Cd007411.

3. Betteridge DJ. What is oxidative stress? Metabolism 2000;49:3-8.

4. Gunes S, Hekim GN, Arslan MA, Asci R. Effects of aging on the male reproductive system. J Assist Reprod Genet. 2016;33:441-54.

5. Smith TB, Baker MA, Connaughton HS, Habenicht U, Aitken RJ. Functional deletion of Txndc2 and Txndc3 increases the susceptibility of spermatozoa to agerelated oxidative stress. Free Radic Biol Med. 2013;65:872-81.

6. Selvaratnam J, Paul C, Robaire B. Male rat germ cells display age-dependent and cell-specific susceptibility in response to oxidative stress challenges. Biol Reprod. 2015;93:72.

7. Bokov A, Chaudhuri A, Richardson A. The role of oxidative damage and stress in aging. Mech Ageing Dev. 2004;125:811-26.
8. Silberstein T, Har-Vardi I, Harlev A, Friger M, Hamou B, Barac T, et al. Antioxidants and polyphenols: concentrations and relation to male infertility and treatment success. Oxid Med Cell Longev. 2016;2016:9140925.

9. Showell MG, Mackenzie-Proctor R, Brown J, Yazdani A, Stankiewicz MT, Hart RJ. Antioxidants for male subfertility. Cochrane Database Syst Rev. 2014:Cd007411.

10. Fan Y, Liu Y, Xue K, Gu G, Fan W, Xu Y, et al. Diet-induced obesity in male C57BL/6 mice decreases fertility as a consequence of disrupted blood-testis barrier. PLoS One. 2015;10:e0120775.

11. Yi R, Deng L, Mu J, Li C, Tan F, Zhao X. The impact of antarctic ice microalgae polysaccharides on d-galactose-induced oxidative damage in mice. Front Nutr. 2021;8:651088.

12. Lai YF, Wang HY, Peng RY. Establishment of injury models in studies of biological effects induced by microwave radiation. Mil Med Res. 2021;8:12.

13. Elangovan N, Chiou TJ, Tzeng WF, Chu ST. Cyclophosphamide treatment causes impairment of sperm and its fertilizing ability in mice. Toxicology 2006;222:60-70.

14. Li C, He X, Huang Z, Han L, Wu X, Li L, et al. Melatonin ameliorates the advanced maternal age-associated meiotic defects in oocytes through the SIRT2-dependent H4K16 deacetylation pathway. Aging (Albany NY) 2020;12:1610-23.

15. Schneider S, Shakeri F, Trötschel C, Arévalo L, Kruse A, Buness A, et al. Protamine2 deficiency initiates a reactive oxygen species (ROS)-mediated destruction cascade during epididymal sperm maturation in mice. Cells. 2020;9:1789.

16. Dai X, Zhang Q, Yu Z, Sun W, Wang R, Miao D. Bmi1 deficient mice exhibit male infertility. Int J Biol Sci. 2018;14:358-68.

17. Ozkosem B, Feinstein SI, Fisher AB, O'Flaherty $C$. Advancing age increases sperm chromatin damage and impairs fertility in peroxiredoxin 6 null mice. Redox Biol. 2015;5:15-23.

18. Selvaratnam JS, Robaire B. Effects of aging and oxidative stress on spermatozoa of superoxide-dismutase 1- and catalase-null mice. Biol Reprod. 2016;95:60.

19. Li D, Meng L, Xu T, Su Y, Liu X, Zhang Z, et al. RIPK1-RIPK3-MLKL-dependent necrosis promotes the aging of mouse male reproductive system. Elife. 2017;6: e27692.

20. Wang $R$, Xue $X$, Wang $Y$, Zhao $H$, Zhang $Y$, Wang $H$, et al. BMI1 deficiency results in female infertility by activating p16/p19 signaling and increasing oxidative stress. Int J Biol Sci. 2019;15:870-81.

21. Zhou SH, Deng YF, Weng ZW, Weng HW, Liu ZD. Traditional chinese medicine as a remedy for male infertility: a review. World J Mens Health. 2019;37:175-85.

22. Commission CP Pharmacopoeia of the People's Republic of China. Beijing: China Pharmaceutical Science and Technology Press; 2015.

23. Xu Q, Bauer R, Hendry BM, Fan TP, Zhao Z, Duez P, et al. The quest for modernisation of traditional Chinese medicine. BMC Complement Alter Med. 2013;13:132.

24. Zhang W, Chen H, Wang Z, Lan G, Zhang L. Comparative studies on antioxidant activities of extracts and fractions from the leaves and stem of Epimedium koreanum Nakai. J Food Sci Technol. 2013;50:1122-9.

25. Li Y, Pham V, Bui M, Song L, Wu C, Walia A, et al. Rhodiola rosea L.: an herb with anti-stress, anti-aging, and immunostimulating properties for cancer chemoprevention. Curr Pharm Rep. 2017;3:384-95.

26. Yu S, Chen K, Li S, Zhang K. In vitro and in vivo studies of the effect of a Chinese herb medicine on osteoclastic bone resorption. Chin J Dent Res. 1999;2:7-11.

27. Sayed SMA, Siems K, Schmitz-Linneweber C, Luyten W, Saul N. Enhanced healthspan in caenorhabditis elegans treated with extracts from the traditional chinese medicine plants Cuscuta chinensis Lam. and Eucommia ulmoides Oliv. Front Pharm. 2021;12:604435.

28. Chen M, Hao J, Yang Q, Li G. Effects of icariin on reproductive functions in male rats. Molecules 2014;19:9502-14.

29. Yuan $D$, Wang $H$, He H, Jia $L, H e ~ Y$, Wang $T$, et al. Protective effects of total flavonoids from Epimedium on the male mouse reproductive system against cyclophosphamide-induced oxidative injury by up-regulating the expressions of SOD3 and GPX1. Phytother Res. 2014;28:88-97.

30. Sze SC, Tong Y, Ng TB, Cheng CL, Cheung HP. Herba Epimedii: anti-oxidative properties and its medical implications. Molecules 2010;15:7861-70.

31. Dastig S, Nenicu A, Otte DM, Zimmer A, Seitz J, Baumgart-Vogt E, et al. Germ cells of male mice express genes for peroxisomal metabolic pathways implicated in the regulation of spermatogenesis and the protection against oxidative stress. Histochem Cell Biol. 2011;136:413.

32. Weir CP, Robaire B. Spermatozoa have decreased antioxidant enzymatic capacity and increased reactive oxygen species production during aging in the Brown Norway rat. J Androl. 2007;28:229-40.

33. Jiang $H$, Zhu WJ, Li J, Chen QJ, Liang WB, Gu YQ. Quantitative histological analysis and ultrastructure of the aging human testis. Int Urol Nephrol. 2014;46:879-85.

34. Hara E, Smith R, Parry D, Tahara H, Stone S, Peters G. Regulation of p16CDKN2 expression and its implications for cell immortalization and senescence. Mol Cell Biol. 1996;16:859-67. 
35. Rayess H, Wang MB, Srivatsan ES. Cellular senescence and tumor suppressor gene p16. Int J Cancer. 2012;130:1715-25.

36. Takahashi A, Ohtani N, Yamakoshi K, lida S, Tahara H, Nakayama K, et al. Mitogenic signalling and the p16INK4a-Rb pathway cooperate to enforce irreversible cellular senescence. Nat Cell Biol. 2006;8:1291-7.

37. Reagan-Shaw $S$, Nihal $M$, Ahmad N. Dose translation from animal to human studies revisited. FASEB J. 2008;22:659-61.

38. Ahmed EA, de Rooij DG. Staging of mouse seminiferous tubule cross-sections. Methods Mol Biol. 2009;558:263-77.

39. Zheng M, Chen X, Cui Y, Li W, Dai H, Yue Q, et al. TULP2, a new RNA-binding protein, is required for mouse spermatid differentiation and male fertility. Front Cell Dev Biol. 2021;9:623738.

40. de Siqueira Bringel $S$, de Amorim Júnior AA, Amorim MJ, Brito LT, Morais RN, de Torres SM, et al. Endocrine and testicular changes induced by olanzapine in adult Wistar rats. J Appl Toxicol. 2013;33:24-31.

41. Selvaratnam J, Robaire B. Overexpression of catalase in mice reduces age-related oxidative stress and maintains sperm production. Exp Gerontol. 2016;84:12-20.

42. Beattie MC, Chen H, Fan J, Papadopoulos V, Miller P, Zirkin BR. Aging and luteinizing hormone effects on reactive oxygen species production and DNA damage in rat Leydig cells. Biol Reprod. 2013;88:100.

43. Xu G, Li JY. CDK4, CDK6, cyclin D1, p16(INK4a) and EGFR expression in glioblastoma with a primitive neuronal component. J Neurooncol. 2018;136:445-52.

44. Sherr CJ, Beach D, Shapiro GI. Targeting CDK4 and CDK6: from discovery to therapy. Cancer Discov. 2016;6:353-67.

45. Wang J, Wong YK, Liao F. What has traditional Chinese medicine delivered for modern medicine? Expert Rev Mol Med. 2018;20:e4.

46. Barati $E$, Nikzad H, Karimian M. Oxidative stress and male infertility: current knowledge of pathophysiology and role of antioxidant therapy in disease management. Cell Mol Life Sci. 2020;77:93-113.

47. Bisht $S$, Faiq $M$, Tolahunase $M$, Dada R. Oxidative stress and male infertility. Nat Rev Urol. 2017;14:470-85.

48. Abdelaziz AS, Kamel MA, Ahmed Al, Shalaby SI, El-Darier SM, Magdy Beshbishy A, et al. Chemotherapeutic potential of epimedium brevicornum extract: The cGMPspecific PDE5 inhibitor as anti-infertility agent following long-term administration of tramadol in male rats. Antibiotics (Basel). 2020;9:318.

49. Sun $M$, Yin Y, Wei J, Chen $X$, Ouyang $H$, Chang $Y$, et al. Development and validation of a HPLC-MS/MS method for simultaneous determination of twelve bioactive compounds in epimedium: application to a pharmacokinetic study in rats. Molecules. 2018;23:1322.

50. Wang Z, Wang D, Yang D, Zhen W, Zhang J, Peng S. The effect of icariin on bone metabolism and its potential clinical application. Osteoporos Int. 2018;29:535-44.

51. Sakkas D, Ramalingam M, Garrido N, Barratt CL. Sperm selection in natural conception: what can we learn from Mother Nature to improve assisted reproduction outcomes? Hum Reprod Update. 2015;21:711-26.

52. Jeyendran RS, Caroppo E, Rouen A, Anderson A, Puscheck E. Selecting the most competent sperm for assisted reproductive technologies. Fertil Steril. 2019;111:851-63.

53. Varghese AC, Sinha B, Bhattaccharyya AK. Current trends in evaluation of sperm function: in vitro selection and manipulation of male gametes for assisted conception. Indian J Exp Biol. 2005;43:1023-31.

54. Srinivas US, Tan BWQ, Vellayappan BA, Jeyasekharan AD. ROS and the DNA damage response in cancer. Redox Biol. 2019;25:101084.

55. Wang JYJ. Cell death response to DNA damage. Yale J Biol Med. 2019;92:771-9.

56. Tower J. Programmed cell death in aging. Ageing Res Rev 2015;23:90-100. Pt A

57. Stobezki R, Titus S, Halicka D, Darzynkiewicz Z, Oktay K. Declining BRCA-mediated DNA repair in sperm aging and its prevention by sphingosine-1-phosphate. Reprod Sci. 2020;27:940-53.

58. Birch J, Gil J. Senescence and the SASP: many therapeutic avenues. Genes Dev. 2020;34:1565-76

59. Krishnamurthy J, Torrice C, Ramsey MR, Kovalev Gl, Al-Regaiey K, Su L, et al. Ink4a/ Arf expression is a biomarker of aging. J Clin Invest. 2004;114:1299-307.
60. Tsygankov D, Liu Y, Sanoff HK, Sharpless NE, Elston TC. A quantitative model for age-dependent expression of the p16INK4a tumor suppressor. Proc Natl Acad Sci USA. 2009;106:16562-7.

\section{ACKNOWLEDGEMENTS}

Pex3 and P16-KO mice were gifts from the research teams of Sha Jiahao and Miao Dengshun from the State Key Laboratory of Reproductive Medicine, Nanjing Medical University. This work was supported by the National Natural Science Foundation of China to Hui Wang (no. 81774075), and a Project Funded by State Key Laboratory of Reproductive Medicine, Nanjing Medical University to Hui Wang.

\section{AUTHOR CONTRIBUTIONS}

H.Y.Z., T.T.Z., Q.Q.H., and H.W. performed the experiments. H.Y.Z., J.H.Y., and Y.Y.P. performed the data analysis. H.Y.Z., T.T.Z. wrote the paper. H.W. and Y.Q. designed and conceptualized the study.

\section{ETHICS STATEMENT}

All animal protocols were approved by the Animal Care and Use Committee of Nanjing Medical University (IACUC-1709008).

\section{COMPETING INTERESTS}

The authors declare no competing interests.

\section{ADDITIONAL INFORMATION}

Supplementary information The online version contains supplementary material available at https://doi.org/10.1038/s41419-021-04435-8.

Correspondence and requests for materials should be addressed to Hui Wang or Yun Qian.

Reprints and permission information is available at http://www.nature.com/ reprints

Publisher's note Springer Nature remains neutral with regard to jurisdictional claims in published maps and institutional affiliations.

Open Access This article is licensed under a Creative Common Attribution 4.0 International License, which permits use, sharing, adaptation, distribution and reproduction in any medium or format, as long as you give appropriate credit to the original author(s) and the source, provide a link to the Creative Commons license, and indicate if changes were made. The images or other third party material in this article are included in the article's Creative Commons license, unless indicated otherwise in a credit line to the material. If material is not included in the article's Creative Commons license and your intended use is not permitted by statutory regulation or exceeds the permitted use, you will need to obtain permission directly from the copyright holder. To view a copy of this license, visit http://creativecommons. org/licenses/by/4.0/.

(c) The Author(s) 2022 